\title{
Bacteria flora associated with different body parts of hatchery reared juvenile Penaeus monodon, tanks water and sediment
}

\begin{abstract}
Bacteria flora of intestine and hepatopancreas, body surface and muscles of juvenile Penaeus monodon along with its rearing water and sediment was analyzed. Juvenile shrimp were reared in four tanks in the Hatchery complex, Department of Aquaculture, Faculty of Agriculture, University Putra Malaysia. Water quality parameters were measured every day. Samples were collected aseptically and homogenized before being inoculated in Tryptone Soy agar, Thiosulphate Citrate Bile Salt agar, MacConkey agar and Pseudomonas-isolating agar. There was no significant difference between water quality parameters and shrimp body weight of replicate tanks. Total plate count for water and total Vibrio count for rearing water and digestive system were within previous reported ranges. Eight different genera were isolated in which 7 genera were identified. Gram negative bacteria were dominant $(72 \%)$ Vibrio was the most dominant genera followed by Shewanella and Burkholderia. Clavibacter followed by Staphylococcus were the most dominant gram positive bacteria. No coliform bacteria was detected in the shrimp body parts and rearing environment. Incidence of Shewanella in the digestive system was significantly higher than sediment, rearing water and muscles. This may be implied its ability to colonize in the digestive tract of juvenile P. monodon.
\end{abstract}

Keyword: Shrimp; Penaeus monodon; Microflora; Vibrio; Shewanella 